\title{
Effects of verbal encouragement on force and electromyographic activations during handgrip exercise
}

\section{Chama Belkhiria ${ }^{1}$, Giovanni de $\operatorname{Marco}^{1,2}$ \& Tarak Driss ${ }^{1,2}$}

\section{Authors affiliations}

${ }^{1}$ Laboratoire CeRSM (EA 2931), Equipe «Analyse du Mouvement en Biomécanique, Physiologie et Imagerie ». UFR STAPS, Université Paris Ouest Nanterre La Défense, 200 avenue de la République, 92000 Nanterre, France.

${ }^{2}$ COMUE Université Paris Lumières, France

Address and contact information of the first author:

Chama Belkhiria, Université Paris Nanterre, UFR STAPS, CeRSM (EA 2931), 200

avenue de la république 92000 Nanterre, France

E-mail: belkhiriachema@hotmail.fr

\section{Conflicts of interest:}

The authors of this manuscript declare that they have no conflicts of interest to disclose.

\section{Funding}

None declared.

\section{Acknowledgements}

We would like to thank the participants that gave their time to take part in this study and

everybody who has contributed to its realization. This work was partly supported by the COMUE Université Paris Lumières. 


\section{Abstract}

Background: The aim of the present study was to examine the effects of verbal encouragement on isometric force and associated electromyographic (EMG) parameters during a handgrip task.

Methods: Twenty-three participants (12 women and 11 men) performed maximal voluntary isometric handgrip contractions following three conditions: 1) verbal encouragement (VE) condition: participants executed isometric contractions while being verbally encouraged. 2) non verbal encouragement (nVE) condition: the same starting and stopping signal but without encouragement. 3) non concentration and non motivation (nCM) condition: self-initiated contractions without concentration and motivation. Start and stop of the contraction were self-initiated. The maximal voluntary force (MVF) and the maximal rate of force development (MRFD) were measured. Integrated EMG corresponding to MVF (iEMGMVF) and to MRFD (iEMG $\mathrm{MRFD}_{\text {) were }}$ collected from Flexor Digitorum Superficialis (FDS) and Extensor Digitorum Communis (EDC) muscles.

Results: MVF was higher during VE compared with nVE $(+11.7 \% ; \mathrm{P}<0.05)$ and $\mathrm{nCM}$ $(+23.2 \% ; \mathrm{P}<0.05)$ conditions. Likewise, MRFD was significantly higher during VE, compared with $\mathrm{nVE}(+21.7 \% ; \mathrm{P}<0.05)$ and $\mathrm{nCM}(+55.4 \% ; \mathrm{P}<0.05)$ conditions. iEMGMvE increased for FDS and EDC during VE, compared to nVE $(+26.19 \%$, $+20.5 \%)$ and $\mathrm{nCM}$ conditions $(+68.85 \%,+48.91 \%)$, respectively. $\mathrm{EEMG}_{\mathrm{MRFD}}$ increased for FDS and EDC during VE, compared to $\mathrm{nVE}(+21.2 \%,+46.07 \%)$ and $\mathrm{nCM}$ conditions $(+23.79 \%,+42.32 \%)$. Furthermore, the reproducibility of all these indices was higher with VE condition. 
Conclusion: Taken together, force production (MVF and MRFD) and EMG data supported the view that muscles activity is considerably influenced by the verbal encouragements during isometric force exercise.

Key words: Verbal encouragement, MVF, MRFD, EMG, Reproducibility

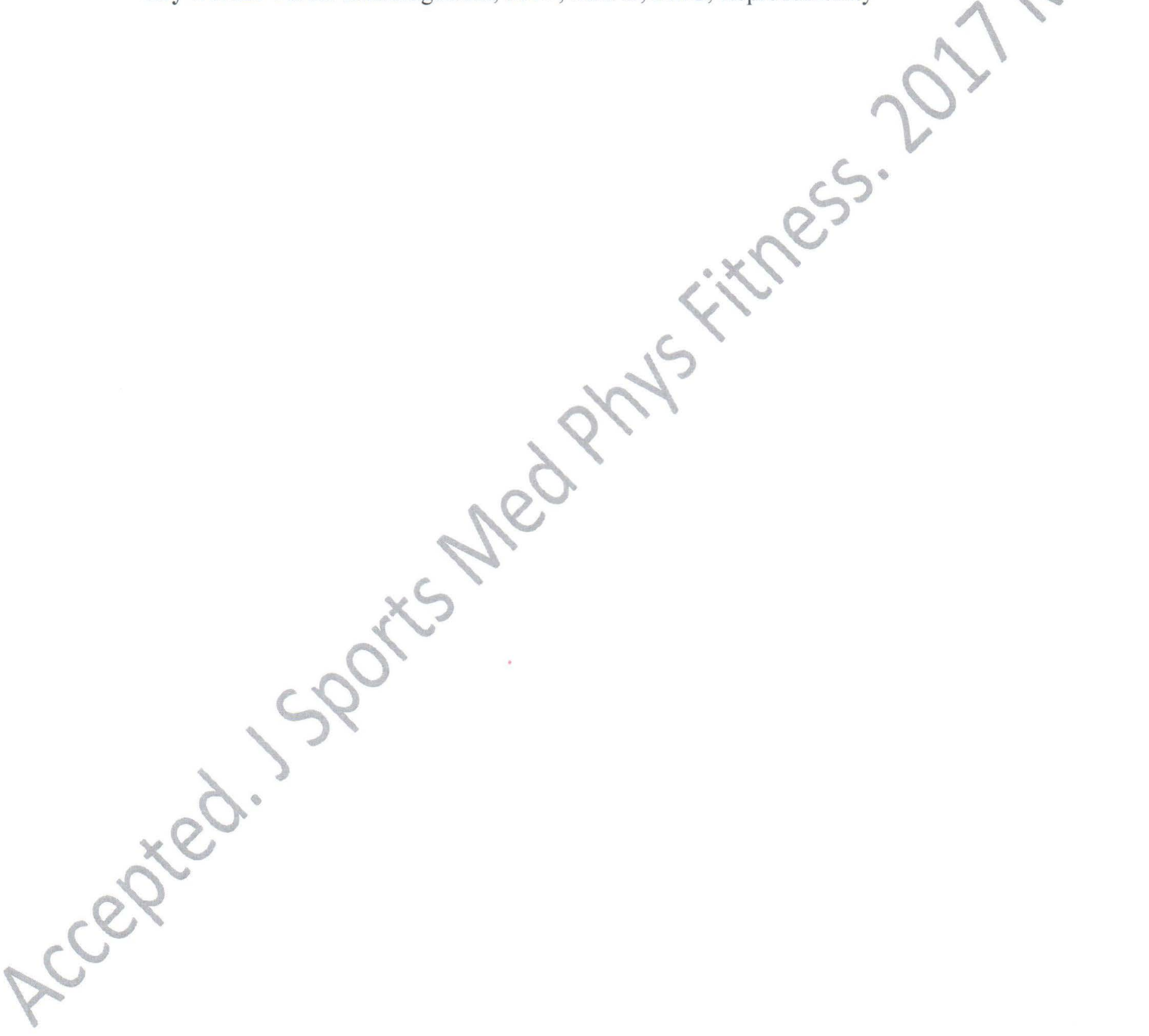




\section{Introduction}

Many previous studies have demonstrated that muscular explosive force production during maximal voluntary contraction depends on histological, morphological and biomechanical factors, such as muscle typology ${ }^{1}$, muscle size and architecture ${ }^{2}$, anatomic muscle cross-sectional area and volume ${ }^{3}$, synchrony of activation onset ${ }^{4}$, musculo-tendinous stiffnerss ${ }^{5}$, recruitment of motor units and the modulation of their firing ${ }^{6}$ and the mechanical properties of the myotendinous complex ${ }^{7-9}$. Moreover, the kind of instruction given by the investigator is also known to influencethe modulation of motor unit activity during explosive force production ${ }^{10-13}$. Indeed, the effect of instruction has largely been studied in small muscle groups with a large cortical representation (somatotopic map), which are involved in precise voluntary movements. For example, Bemben et al. ${ }^{14}$ and Sahaly et al. ${ }^{13-15}$ studied the effect of instruction during isometric exercises on MVF, MRFD and EMG. MRFD was significantly higher when subjects were instructed to exert the most explosive force by concentrating on the fastest contraction without concern for achieving maximal force than when they were asked to exert muscle force with hard-and-fast instruction ${ }^{13,15}$. Explosive muscular force production expressed by MVF and MRFD is considered to be important in many sport-specific and functional daily tasks. MVF and MRFD are involved in the performance of sporting movements, such as jumping ${ }^{16-18}$, and in providing dynamic joint stability and ligament protection ${ }^{19}$. The rate of force development should also depend on nervous factors. It has been demonstrated that MRFD is lower during voluntary contractions than during electrical stimulations ${ }^{20,21}$. Furthermore, since the pioneering study by Adrian and Bronk ${ }^{22}$, it is well known that the gradation of muscle force is the result of an increasing firing rate and additional motor unit recruitment. 
Among studies analyzing the instruction effect, the use of positive VE has been shown to improve motivation and motor performance in various activities, such as voluntary isometric contractions ${ }^{23-25}$. VE represents a cognitive and motivational process, which is reflected in a large, distributed bilateral cerebro-cerebellar network ${ }^{26}$. According to McNair et al. ${ }^{23}$, VE leads to suppression of the supraspinal inhibition, thus resulting in enhancement of muscle strength. Furthermore, Belanger and McComas ${ }^{6}$ suggested that the activation of motor units may be inhibited when a maximal voluntary contraction is required due to a supraspinal drive acting on the motor units. In a recent functional Magnetic Resonance Imaging (fMRI) study, Belkhiria et al. ${ }^{26}$ found that mean handgrip force measured during VE $(29.26 \mathrm{~kg} ; \mathrm{p}<0.05)$ was significantly higher relative to the non-verbal condition $(26.97 \mathrm{~kg} ; \mathrm{P}=0.004)$. The motivation induced by VE was maintained thanks to a closed-connectivity loop between cerebral and cerebellar cortex specifically through the red nucleus and striatal network ${ }^{26}$. Furthermore, Generating a maximum muscle tension involves not only the recruitment of motor units, but also a modulation of their firing rates ${ }^{6}$ by the brain networks, reinforced by the type of VE given to the participant during the contraction ${ }^{26}$. However, these VE works did not study at any time the effect of VE at specific and crucial force instants such as MVF, MRFD or associated EMG. Collectively, these findings indicated that VE is known to influence cerebral activity, and thus may lead one to suggest that VE could affect the neuromuscular activation (force and associated electromyography).

From a methodological perspective, one also has to consider the relative advantages and limits of previous cited studies. Whereas verbal encouragement previous studies revealed neuromuscular increases and yielded a high specificity, their efficiency may be limited by the selection of only large time intervals and the lack of time accuracy, and 
reproducibility. Independent of methodological differences, interpretation of the current literature thus faces the standing that VE effect is present in several neuromuscular activation methods but absent in the specific and crucial force and iEMG instants. In order to provide a precise identification of $\mathrm{VE}$ effect, this study aimed to: i) determine how VE could influence force parameters during high accuracy and crucial force time intervals such as MVF and MRFD; ii) determine whether the EMG activity might be substantially responsive to VE effect; iii) assess how the reproducibility of MVF, MRFD and EMG could depend on VE. To address these aims, we compared MVF, MRFD and EMG across three diverse handgrip conditions: $\mathrm{VE}, \mathrm{nVE}$ and $\mathrm{nCM}$. It was hypothesized that VE condition, acting as a motivational factor, would result in higher MVF and MRFD parameters. This increase would be explained by neuromuscular activation expressed by iEMG activity of solicited muscles.

\section{Materials and methods}

\section{Participants}

Twenty-three active young adults (12 active women and 11 active men, aged $25.1 \pm 5.9$ years ranging from 18 to 32 years) participated in this experiment. All of them were right-handed and volunteers. They had to strongly concentrate on the movement to be performed of pressing a handgrip as hard as possible without making any sudden movements. For the assessment of inclusion and exclusion criteria, a questionnaire was used to examine the participant's health condition. None of them practiced intense or isometric activities of upper or lower limbs (e.g., body building, climbing, weightlifting...). They have no neuromuscular or cardio-respiratory disease. Female participants were not pregnant or lactating. Participants have not a history of alcohol or recreational drug abuse within 12 months prior to the study. Participants were 
asked to respond to the questionnaire before the beginning of the experiment under supervision of a study nurse to ensure accurate understanding of the questions. All questions were answered with "yes" or "no," including space for additional comments. All participants were healthy and had no known neuromuscular disorders at the time of the study. They have given their informed consent for participation in this research study. The study was approved by the Institutional Review Board, and was conducted according to the guidelines of the Declaration of Helsinki.

\section{Protocol}

The protocol began with a familiarization and warm-up phase with the handgrip device. Participants were lying on their backs with connected forearm EMG electrodes and with headphones placed on their ears. They were asked to repeat the following sequence five times: mental preparation, then motor execution by squeezing a handgrip as hard as possible, and afterwards rest. In this paper, we only focus on the execution period of the three following randomized conditions:

i) Verbal Encouragement (VE) condition: participants achieved a preparation period of 6.6 seconds and then they received a starting and stopping contraction signal (Go/Stop). During the contraction period of 4.4 seconds, participants were verbally encouraged by a recorded human voice repeating firmly, "Go Go Go..." 20 times to encourage them to squeeze the handgrip as hard as possible. Finally, a long rest period of 44 seconds allowed participants to recover effort. The verbal encouragement was recorded by WavePad Audio Editing Software.

ii) Non-verbal encouragement (nVE) condition: It includes exactly the same steps of VE condition but without verbal encouragement during contraction movement. 
iii) Non concentration and non motivation $(\mathrm{nCM})$ condition: the start and stop of contractions were self-initiated by the participants themselves. They heard a beep every 55 seconds (five times) to remind them to squeeze the handgrip as hard as possible during 4.4 seconds. The participants had to prepare and to squeeze the handgrip when they felt ready without motivation and concentration.

This protocol was tested in a precedent fMRI study (14) and the used experimental periods were multiples of 2.2 seconds, which is a time repetition parameter needed in fMRI measures. Both studies used the same paradigm with different hypotheses and they are completely independent.

\section{Force data acquisition}

The isometric contraction (duration 4.4 seconds) was measured by a pressure captor (hand dynamometer TSD121B-MRI, Biopac Systems Inc., Santa Barbara, CA) connected to the Biopac system MP150 (Biopac MP150, Systems Inc., Santa Barbara, CA). The handgrip device was held by the right hand of the participant. The transducer was connected to an amplifier DA100C (Biopac MP150, System Inc., Santa Barbara, CA), whose output was directed to the AcqKnowledge software (Version 4.2, System Inc., Santa Barbara, CA). This amplifier recorded the data on the hard drive of a personal computer. The sampling frequency of the force signal was digitized at 1000 $\mathrm{Hz}$

\section{EMG data acquisition}

Simultaneously with force data measurements, electromyographic signals were digitized and recorded on the same computer. The flexor digitorum superficialis (FDS) and extensor digitorum communis (EDC) muscles of the right forearm were identified by palpating the skin when participants flexed and extended their fingers. The overlying 
skin was shaved and rubbed with an alcohol wipe to remove dead cells, dirt or skin oils. Bipolar electrodes (ADD208, 8-mm recording diameter, System Inc., Santa Barbara, CA) were firmly attached on the skin surface overlying each of the two muscles. A reference electrode was placed on the skin overlying the lateral epicondyle near the elbow joint of the right arm. Surface EMG signals were recorded using a sampling rate of 1,000 Hz. The EMG signal was amplified (gain range at 2000) and filtered using a band-pass filter $(125 \mathrm{~Hz})$.

\section{Force and EMG data processing}

MVF was defined as the highest peak force recorded during one trial. MRFD was equal to the steepest slope calculated in a $20-\mathrm{ms} \mathrm{time} \mathrm{window}^{13}$. The value of MRFD was expressed in newtons per second and in relative units, i.e. as e percentage of MVF per second $\left[\mathrm{MRFD} \%=(\mathrm{MRFD} / \mathrm{MFV})^{*} 100\right]^{13}$. EMG data for both muscles were rectified and integrated (iEMG) during the specific phase and divided by the duration of the phase corresponding to average EMG. The value of iEMG corresponding to MVF (iEMG $\mathrm{MVF}_{\mathrm{F}}$ ) was computed by integrating EMG during a $128-\mathrm{ms}$ window previously to

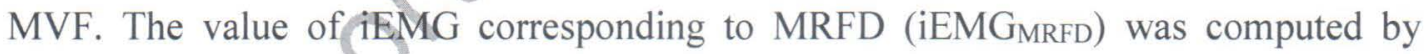
integrating EMG during a 128-ms window around MRFD (64 ms before and $64 \mathrm{~ms}$ after MRFD) $3^{5}$. Five trials have been executed in each condition. For each condition, the mean of the three best trials of MVF and MRFD were averaged to analyze the data.

\section{Statistical analyses}

Statistical analyses were carried out using Statistica 10.0 (StatSoft, Maisons-Alfort, France) and data are presented as mean $( \pm \mathrm{SD})$. The normality was tested using the Shapiro-Wilk's test for all dependent variables. One-way ANOVA with repeated measure and Bonferroni post-hoc test were used to examine the effect of instruction on 
measured variables. Relative changes (\%) in dependent variables are expressed with 95\% confidence interval (95\% CI). The standardized differences or Cohen effect sizes (ES, $95 \% \mathrm{CI}$ ) of differences in measured variables between the conditions were calculated using the pooled standard deviation ${ }^{27}$. Threshold values for Cohen ES statistics were $0-<0.20$ (trivial), $0.2-<0.50$ (small), $0.50-<0.80$ (medium), $\geq 0.80$ (large) 28. Person Correlation Coefficient was used to correlate MFV with MRFD. The intraparticipant coefficient of variation in each condition $(\mathrm{CV} \%)$ of the three best trials of MVF and MRFD and the associated EMGMVF and EMGMRFD activities were examined using two-way ANOVA with repeated measures and a Bonferroni post-hoc test. All significance thresholds were set at $\mathrm{P}<0.05$.

\section{Results}

\section{MVF and MRFD}

The mean $( \pm \mathrm{SD})$ values of MVF, MRFD and MRFD \% for each condition were presented in Table 1. A significant main effect of instruction was found for all force data (Table 1). The Bonferroni Post-hoc test showed that the values of MVF, MRFD, and MRFD\% were significantly higher with VE compared with nVE and nCM. There were significant correlations between MVF and MRFD for each instruction $0.82 \leq \mathrm{r} \leq$ $0.84(\mathrm{P}<0.001)$ for $\mathrm{nCM}, \mathrm{nVE}$ and $\mathrm{VE}$, respectively. There were no significant differences between instructions for both CV\% MVF and CV\% MRFD. However, CV\% MRFD was significantly higher than CV\% MVF.

\section{EMG activity}

The mean $( \pm \mathrm{SD})$ values of iEMG for MVF and MRFD were presented in Table 2. A significant main effect of instruction was found for EMG data (Table 2). The value of 
iEMGMVF was significantly higher in $\mathrm{VE}$ than in $\mathrm{nVE}$ and $\mathrm{nCM}$ for all participants for FDS and EDC. Similar significant results were also found for iEMGMRFD.

The mean $( \pm \mathrm{SD})$ values of coefficient of variation of iEMG for MVF and MRFD were presented in Table 3. The CV\% of EMGMRFD was significantly higher than $\mathrm{CV} \%$ of $\mathrm{EMG}_{\mathrm{MVF}}$ only during $\mathrm{nCM}$ for EDC muscle. $\mathrm{CV} \%$ of $\mathrm{EMG}_{\mathrm{MVF}}$ and $\mathrm{EMG}_{\mathrm{MRFD}}$ for both FDS and EDC muscles were significantly higher with VE.

Standardized differences between instructions were presented in table 4. Taken together, these results indicated supplementary significant differences between the three instructions. For all tested parameters, the verbal encouragement effect was higher compared to both $\mathrm{nVE}$ and $\mathrm{nCM}$.

\section{Discussion}

This study investigated for the first time the verbal encouragement effect during handgrip exercise by the combination of force production and EMG analysis. In contrast to previous instruction studies ${ }^{13-15,23}$, we pursued a different approach and found some novelties. Addressing many aims, the present results confirmed our hypothesis and indicated that: i) verbal encouragement increased significantly MVF and MRFD; (ii) iEMG activity was substantially related to VE; iii) VE had positive and significant effect on EMG reproducibility; iiii) VE would decrease intra-subject MRFD variability.

The previous researches reported VE increases only during large time intervals such as during aerobic Wingate test ${ }^{29}, \mathrm{VO} 2$ max and blood lactate concentration during a treadmill test ${ }^{30}$, muscular endurance ${ }^{31}$, elbow flexors during an isometric muscle action ${ }^{23}$, knee extension ${ }^{24}$, and triceps surae flexion ${ }^{25}$. An advantage result of the present 
study was the concurrent dependence of both MVF and MRFD on VE, as suggested by the comparison to $\mathrm{nVE}$ and $\mathrm{nCM}$ conditions (Table 4). This VE effect on MRFD was observed on the different indices of rate of force development: maximal slope in absolute values and relative MRFD. Increases in both absolute and relative values of rate of force development with VE condition implied a decrease in the time to develop a specific level of force output. The positive correlation between MVF and MRFD for each instruction confirmed that MRFD expressed in absolute values depends on maximal strength ${ }^{32}$, in addition to shortening velocity, skeletal muscle compliance and muscle activation. Moreover, it was confirmed that the rapid force production is closely related to central parameters ${ }^{33}$. More specifically, RFD can be strongly affected by both the supraspinal drive and presynaptic inhibition 33 . Gandevia ${ }^{34}$ suggested that diverse spinal and supraspinal mechanisms influence the supraspinal drive and/or motor units firing rate. In support of previous studies ${ }^{35-38}$, whatever the instruction, coefficients of variation and consequently intra-subjects variabilities were significantly higher in MRFD than MVF.

To the best of our knowledge, this study is the first to evaluate VE effect on neuromuscular activation during explosive voluntary contractions. Maximal contraction data showed greater iEMG in FDS and EDC muscles during VE condition when compared with nVE and nCM conditions (Tables 2, 4). Current literature suggested that this increase might be partly related to voluntary drive to human skeletal muscles through changes in the spinal and peripheral transmission of the neural drive ${ }^{39}$. The increased iEMGs are synonymous with increases in specific neural drive, including increases in motor unit recruitment and/or firing frequency ${ }^{40,41}$ driven by the spinal and/or supraspinal centre ${ }^{42-44}$. Our results indicated that the abilities of the central nerve 
system to activate and recruit motor units of the FDS and EDC muscles, including early recruitment and greater motor unit synchronisation, were significantly increased during maximal voluntary contractions associated to VE. According to McNair et al. ${ }^{23}$, VE may lead to a disengagement of the supraspinal inhibition, thus resulting in an enhancement of muscle strength. Moreover, our results corroborate with Lieber's explanation ${ }^{45}$ stating that the greater the number of motor units recruited through a stimulus, the greater the resultant muscle generated will also be. The iEMG data explain the highest performances of both MVF and MRFD but do not explain the higher increase of MRFD compared to MVF.

Moreover, the important electric activity of EDC during VE condition revealed a significant effect of the instruction on antagonist muscle during isometric contraction. One possible explanation for this result is that, in humans, the forearm extensor offers static stability of the elbow wrist joint. For example, the role of the extensor carpi ulnaris (similar to EDC) is to control or resist wrist flexion ${ }^{46}$. The extensor muscles also act to generate large and dynamic torque through a high rate of afferent or efferent activation. The primary functional role of the extensor muscle is to open the hand, preparing for manual manipulations during both finest control and power grip ${ }^{47}$. It has been demonstrated that the amplitude of EDC-EMG was much higher than the amplitude of FDS-EMG ${ }^{48}$.

Explosive strength during a fast contraction is determined not only by muscle contraction speed-related characteristics but also by neural factors, as classically inferred from the rate of EMG rise (i.e., the EMG counterpart of RFD), especially in the earlier time intervals of the contraction (i.e., $<100 \mathrm{~ms}$ ) ${ }^{49,50}$. In support of this notion, previous studies proposed that iEMG changes during muscle contraction offer a 
combined measure of the number of active fibres and their frequency of excitation ${ }^{51,52}$. These observations suggested that EMG data in response to VE might partly be due to the facilitation of the recruitment of large, fast motor units, which are expected to be recruited only at higher forces, increasing motor units firing rates, and a greater degree of motor units synchronization. Consequently, the current results corroborated with various pathological investigations ${ }^{53,54}$ reporting that a high degree of motivation is an important factor to obtain higher EMG activity. These studies provided encouraging verbal feedback to help focus the patient's attention and increase motivation. Thus, our results associated to physical and sports activities as well as pathological studies suggest that the ability to produce higher EMG activity associated to force parameters depends on motivational factors (e.g. verbal encouragement) as well as the increase of muscle activation at the onset of the contraction.

The results of the present study highlight the global reproducibility during isometric exercise associated to VE. The coefficient of variation of EMG was higher in MRFD compared with MVF (Table 3 ) as observed in the previous studies ${ }^{55}$.

From neuronal side, in a previous fMRI handgrip study ${ }^{26}$, verbal encouragement reinforced the activity of brain regions (e.g. primary motor cortex, dorsolateral prefrontal cortex, orbitofrontal cortex, superior temporal gyrus and lobule VI of cerebellum). Specifically, the anterior part of the right cerebellum lobule VI was activated by motor execution, while its posterior part was activated by VE. More specifically, VE increased constituted a closed connectivity between cerebral and cerebellar through the red nucleus and striatal network.

Notwithstanding, this study has some limitations. The main one was the restriction of our EMG analysis only to the motor execution stage. The EMG treatment focused only 
on high EMG magnitudes and not on low magnitudes proper to the preparation phase. Further study is needed to develop EMG descriptors during preparation stage in order to understand the relationship between motor preparation and execution tasks during verbal encouragement for both agonist and antagonist muscles. It would be interesting to demonstrate how the complementarities of these force indices could allow to better understand temporally the underlying of neurophysiological and muscular mechanisms associated with cerebral activity. Based on the literature and on the somatotopic organization of the sensorimotor and motor cortex, it would be interesting to study the relationship between the neuromuscular parameters FMV, MRFD and EMG and the corticospinal neuronal transmission from motor cortex to the forearm muscles. This relationship could be measured by using electroencephalography based on the analysis of the cortico-muscular coherence of the beta bands during an isometric force contraction task.

\section{Conclusion}

Taking into account the VE condition on MVF, MRFD and EMG, the present study might provide the potential motivational basis of how the neuromuscular system and less intra-participant variability in MVF compared to MRFD and the decrease of intraparticipant variability in MRFD. It seems reasonable to assume that VE improves the reproducibility of the different measured parameters. The constant encouragements effeets associated to motivational system might provide the necessary process here. The good agreement between anatomical motivational and motor areas and their respective functional roles provide strong evidence for their mutual involvement in specific neural networks during VE. Finally, these results imply that it is important for researchers investigating the improvement of neuromuscular parameters and human motor 
performance to focus on verbal encouragement, particularly for the exploration of explosive force production.

\section{Titles of tables}

Table 1: Force and rate of force development for each condition

Table 2: EMG activity for each condition for FDS and EDC

Table 3: Coefficient of variation of EMGMVF and EMGMRFD for each condition

Table 4: Standardized differences between instructions and percent chances that the true differences were higher/similar/lower

\section{References}

1. Enoka RM, Duchateau J. Inappropriate interpretation of surface EMG signals and muscle fiber characteristics impedes understanding of the control of neuromuscular function. Journal of Appiedl Physiology. 2015;119(12):15161518 .

2. Erskine RM, Fletcher G, Folland JP. The contribution of muscle hypertrophy to strength changes following resistance training. European Journal of Applied physiology. 2014:114(6):1239-1249.

3. Harridge SD, Bottinelli R, Canepari M, et al. Whole-muscle and single-fibre contractile properties and myosin heavy chain isoforms in humans. Pflugers Archiv : European Journal of Physiology. Sep 1996;432(5):913-920.

4. Tillin NA, Jimenez-Reyes P, Pain MT, Folland JP. Neuromuscular performance of explosive power athletes versus untrained individuals. 2010.

5. Waugh CM, Korff T, Fath F, Blazevich AJ. Rapid force production in children and adults: mechanical and neural contributions. Medicine and Science in Sports and Exercise. 2013;45(4):762-771.

Belanger A, McComas A. Extent of motor unit activation during effort. Journal of Applied Physiology. 1981;51(5):1131-1135.

7. Bojsen-Moller J, Magnusson SP, Rasmussen LR, Kjaer M, Aagaard P. Muscle performance during maximal isometric and dynamic contractions is influenced by the stiffness of the tendinous structures. Journal of Applied Physiology. Sep 2005;99(3):986-994. 
8. Blazevich AJ, Cannavan D, Horne S, Coleman DR, Aagaard P. Changes in muscle force-length properties affect the early rise of force in vivo. Muscle \& Nerve. 2009;39(4):512-520.

9. Wu YK, Lien YH, Lin KH, Shih TF, Wang TG, Wang HK. Relationships between three potentiation effects of plyometric training and performance. Scandinavian Journal of Medicine \& Science in Sports. 2010;20(1):e80-e86.

10. Bozic PR, Pazin N, Berjan B, Jaric S. Evaluation of alternating consecutive maximum contractions as an alternative test of neuromuscular function. European Journal of Applied Physiology. 2012;112(4):1445-1456.

11. Christ CB, Boileau RA, Slaughter MH, Stillman RJ, Cameron J. The effect of test protocol instructions on the measurement of muscle function in adult women. Journal of Orthopaedic \& Sports Physical Therapy. 1993;18(3):502510 .

12. Holtermann A, Roeleveld K, Engstrøm M, Sand T. Enhanced H-reflex with resistance training is related to increased rate of force development. European Journal of Applied Physiology. 2007;101(3):301-312.

13. Sahaly R, Vandewalle H, Driss T, Monod H. Maximal voluntary force and rate of force development in humans--importance of instruction. European Journal of Applied Physiolog. Aug 2001;85(3-4):345-350.

14. Bemben MG, Clasey JL, Massey BH. The effect of the rate of muscle contraction on the force-time curve parameters of male and female subjects. Research Quarterly for Exercise and Sport. 1990;61(1):96-99.

15. Sahaly R, Vandewalle H, Driss T, Monod H. Surface electromyograms of agonist and antagonist muscles during force development of maximal isometric exercises--effects of instruction, European Journal of Applied Physiolog. Mar 2003;89(1):79-84.

16. Marcora S, Miller MK. The effect of knee angle on the external validity of isometric measures of lower body neuromuscular function. Journal of Sports Sciences. 2000;18(5):313-319.

17. de Ruiter CJ, Van Leeuwen D, Heijblom A, Bobbert MF, de Haan A. Fast unilateral isometric knee extension torque development and bilateral jump height. Medicine and Science in Sports and Exercise. Oct 2006;38(10):18431852.

18. Driss $\mathrm{T}$, Vandewalle $\mathrm{H}$, Monod $\mathrm{H}$. Maximal power and force-velocity relationships during cycling and cranking exercises in volleyball players. Journal of Sports Medicine Physical Fitness. 1998;38:286-293.

19. Shultz SJ, Perrin DH. Using surface electromyography to assess sex differences in neuromuscular response characteristics. Journal of Athletic Training. 1999;34(2):165.

20. Currier DP, Petrilli CR, Threlkeld AJ. Effect of graded electrical stimulation on blood flow to healthy muscle. Physical Therapy. 1986;66(6):937-943.

21. Koryak Y. Electromyographic study of the contractile and electrical properties of the human triceps surae muscle in a simulated microgravity environment. The Journal of physiology. 1998;510(1):287-295.

22. Adrian ED, Bronk DW. The discharge of impulses in motor nerve fibres: Part II. The frequency of discharge in reflex and voluntary contractions. The Journal of Physiology. 1929;67(2):i3. 
23. McNair PJ, Depledge J, Brettkelly M, Stanley SN. Verbal encouragement: effects on maximum effort voluntary muscle: action. British Journal of Sports Medicine. 1996;30(3):243-245.

24. Amagliani RM, Peterella JK, Jung AP. Type of encouragement influences peak muscle force in college-age women. International Journal of Exercise Science. 2010;3(4):165.

25. Binboğa E, Tok S, Catikkas F, Guven S, Dane S. The effects of verbal encouragement and conscientiousness on maximal voluntary contraction of the triceps surae muscle in elite athletes. Journal of Sports Sciences. 2013;31(9):982-988.

26. Belkhiria C, Driss T, Habas C, Jaafar H, Guillevin R, de Marco G. Exploration and Identification of Cortico-Cerebellar-Brainstem Closed Loop During a Motivational-Motor Task: an fMRI Study. The Cerebellum. 2016:1-14.

27. Cohen J. A power primer. Psychological Bulletin. 1992;112(1):155.

28. Cohen J. Statistical Power Analysis for the Behavioral Sciences, 2 ed 1988.

29. Karaba-Jakovljević D, Popadić-Gaćeša J, Grujić N, Barak O, Drapšin M. Motivation and motoric tests in sports. Medicinski pregled. 2007;60(5-6):231236.

30. Andreacci JL, Lemura LM, Cohen SL, Urbansky EA, Chelland SA, Duvillard SPv. The effects of frequency of encouragement on performance during maximal exercise testing. Journal of Sports Sciences. 2002;20(4):345-352.

31. Bickers MJ. Does verbal encouragement work? The effect of verbal encouragement on a muscular endurance task. Clinical Rehabilitation. 1993;7(3):196-200.

32. Hartmann H, Bob A, Wirth K, Schmidtbleicher D. Effects of different periodization models on rate of force development and power ability of the upper extremity. The Journal of Strength \& Conditioning Research. 2009;23(7):1921-1932.

33. Johnson ST, Kipp K, Norcross MF, Hoffman MA. Spinal and supraspinal motor control predictors of rate of torque development. Scandinavian Journal of Medicine \& Seience in Sports. 2015;25(5):623-629.

34. Gandevia S, Spinal and supraspinal factors in human muscle fatigue. Physiological Teviews. 2001;81(4):1725-1789.

35. De Witt JK, English KL, Crowell JB, et al. Isometric Mid-Thigh Pull Reliability and Relationship to Deadlift 1RM. The Journal of Strength \& Conditioning Research. 2017.

36. Driss T, Sahaly R, Vandewalle H, Isère L, Le Pellec-Muller A, Monod H. Effect of instruction on the reliability of maximal voluntary force and maximal rate of force development during isometric contraction. Reproduction Nutrition Development. 2006;46 (3):306-307.

37. Sahaly R. Effet de la consigne sur les indices mécaniques et électromyographiques de la contraction musculaire isométrique, $P h D$ Thesis. Université Pierre \& Marie Curie, Paris 6; 2004.

38. Viitasalo J, Saukkonen S, Komi P. Reproducibility of measurements of selected neuromuscular performance variables in man. Electromyography and Clinical Neurophysiology. 1980;20(6):487. 
39. Arabadzhiev TI, Dimitrov VG, Dimitrov GV. The increase in surface EMG could be a misleading measure of neural adaptation during the early gains in strength. European Journal of Applied Physiology. 2014;114(8):1645-1655.

40. Cannon J, Kay D, Tarpenning KM, Marino FE. Comparative effects of resistance training on peak isometric torque, muscle hypertrophy, voluntary activation and surface EMG between young and elderly women. Clinical Physiology and Functional Imaging. 2007;27(2):91-100.

41. Lepers R, Millet GY, Maffiuletti NA. Effect of cycling cadence on contractile and neural properties of knee extensors. Medicine and Science in Sports and Exercise. 2001;33(11):1882-1888.

42. Gondin J, Duclay J, Martin A. Soleus-and gastrocnemii-evoked V-wave responses increase after neuromuscular electrical stimulation training. Journal of Neurophysiology. 2006;95(6):3328-3335.

43. Reeves ND, Narici MV, Maganaris CN. Strength training alters the viscoelastic properties of tendons in elderly humans. Muscle \& Nerve. 2003;28(1):74-81.

44. Wang H-K, Lin K-H, Wu Y-K, Chi S-C, Shih TT-F, Huang Y-C. Evoked spinal reflexes and force development in elite athletes with middle-portion Achilles tendinopathy. Journal of Orthopaedic \& Sports Physical Therapy. 2011;41(10):785-794.

45. Lieber RL. Skeletal muscle structure, function, and plasticity. Lippincott Williams \& Wilkins; 2002.

46. Gibbons S, Mottram S, Comerford M. Stability and movement dysfunction related to the elbow and forearm. Orthopaedic Division Review. 2001:15-33.

47. Park WH, Li S. Responses of finger flexor and extensor muscles to transcranial magnetic stimulation during isometric force production tasks. Muscle \& Nerve. 2013;48(5):739-744.

48. Claudon L. Evaluation of grip force using electromyograms in isometric isotonic conditions. International Journal of Occupational Safety and Ergonomics. 1998;4(2):169-184.

49. Aagaard P, Simonsen EB, Andersen JL, Magnusson P, Dyhre-Poulsen P. Increased rate of force development and neural drive of human skeletal muscle following resistance training. Journal of Applied Physiology. Oct 2002;93(4):1318-1326.

50. De Ruiter C, Kooistra R, Paalman M, De Haan A. Initial phase of maximal voluntary and electrically stimulated knee extension torque development at different knee angles. Journal of Applied Physiology. 2004;97(5):1693-1701.

51. Bigland B, Lippold O. Motor unit activity in the voluntary contraction of human

* muscle. The Journal of Physiology. 1954;125(2):322.

52. Edwards R, Lippold $O$. The relation between force and integrated electrical activity in fatigued muscle. The Journal of Physiology. 1956;132(3):677.

53. Lourenção MIP, Battistella LR, de Brito CMM, Tsukimoto GR, Miyazaki MH. Effect of biofeedback accompanying occupational therapy and functional electrical stimulation in hemiplegic patients. International Journal of Rehabilitation Research. 2008;31(1):33-41.

54. Doğan-Aslan M, Nakipoğlu-Yüzer GF, Doğan A, Karabay İ, Özgirgin N. The effect of electromyographic biofeedback treatment in improving upper extremity 
functioning of patients with hemiplegic stroke. Journal of Stroke and Cerebrovascular Diseases. 2012;21(3):187-192.

55. Vaconsin M, Driss T, Sahaly R, Vandewalle H, Le Pellec-Muller A. Etude de l'effet de la consigne sur la reproductibilité de la force isométrique et de l'électromyographie de surface. 13th Congress of ACAPS, Lyon, France Approche Pluridisciplinaire de la Motricité Humaine. 2009; 393-394. 
Table 1: Force and rate of force development for each condition

\begin{tabular}{|c|c|c|c|c|c|c|}
\hline & nCM & nVE & VE & $F(2,44)$ & $\eta^{2}$ & $\mathbf{P}$ \\
\hline MVF (kg) & $26.74 \pm 12.36$ & $28.98 \pm 12.45 \#$ & $32.28 \pm 13.76 \& t$ & 44.65 & 0.67 & $<0.001$ \\
\hline $\operatorname{MRFD}\left(\mathrm{kg} \cdot \mathrm{s}^{-1}\right)$ & $120.36 \pm 75.09$ & $147.33 \pm 82.99 \#$ & $182.14 \pm 110.65 \xi \%$ & 28.04 & 0.56 & $<0.001$ \\
\hline $\operatorname{MRFD} \%\left(. \mathrm{s}^{-1}\right)$ & $439.77 \pm 148.36$ & $495.41 \pm 144.56^{*}$ & $542.32 \pm 174.16 \S$ & $11.12=$ & 0.36 & $<0.001$ \\
\hline $\mathrm{CV} \% \mathrm{MVF}$ & $6.61 \pm 5.7$ & $6.2 \pm 3.25$ & $5.74 \pm 3.26$ & & 0.01 & 0.71 \\
\hline CV\% MRFD & $13.8 \pm 7.1 \mathrm{~A}$ & $10.56 \pm 6.1 \mathrm{~A}$ & $10.5 \pm 5.75$ & 2.16 & 0.08 & 0.122 \\
\hline
\end{tabular}

Verbal encouragement (VE), non verbal encouragement (nVE), non concentration and motivation (nCM)

* $(\mathrm{p}<0.05), \#(\mathrm{p}<0.01), \S(\mathrm{p}<0.001)$ significantly higher compared with nCM

$\$(p<0.001)$ significantly higher compared with $n V E$

$¥(\mathrm{p}<0.001)$ significantly higher compared with $\mathrm{VE}$

$\neq(\mathrm{p}<0.001)$ significantly higher compared with $\mathrm{VE}$

A $(p<0.001)$ significantly higher compared with $\mathrm{CV} \% \mathrm{MVF}$ 
Table 2: EMG activity for each condition for FDS and EDC

\begin{tabular}{|c|c|c|c|c|c|c|c|}
\hline & & $\mathrm{nCM}$ & nVE & VE & $F(2,44)$ & $\eta^{2}$ & $P$ \\
\hline \multirow[b]{2}{*}{$\mathrm{iEMG}_{\mathrm{MVF}}(\mu \mathrm{v})$} & FDS & $30.41 \pm 10.36$ & $39.70 \pm 15 \S$ & $\begin{array}{l}48.87 \pm \\
17.938 t\end{array}$ & 27.645 & 0.56 & 20.001 \\
\hline & EDC & $27.86 \pm 8.63$ & $32.78 \pm 9.04 \S$ & $\begin{array}{l}39.15 \pm \\
12.81 \S t\end{array}$ & & 0.52 & $<0.001$ \\
\hline \multirow{2}{*}{$\mathrm{iEMG}_{\mathrm{MRFD}}(\mu v)$} & FDS & $28.88 \pm 8.80$ & $34.05 \pm 8.27 \S$ & $\begin{array}{l}41.08 \pm \\
10.93 \S t\end{array}$ & & 0.66 & $<0.001$ \\
\hline & EDC & $29.87 \pm 5.64$ & $34.7 \pm 7.38 \S$ & $42.03 \pm 7.42$ & 66.735 & 0.75 & $<0.001$ \\
\hline
\end{tabular}

Verbal encouragement (VE), non verbal encouragement (nVE), non concentration and motivation (nCM)

$\S(\mathrm{p}<0.001)$ significantly higher compared with $\mathrm{nCM}$

$\ddagger(\mathrm{p}<0.001)$ significantly higher compared with $\mathrm{nVE}$ 
Table 3: Coefficient of variation (CV\%) of EMGMVF and $\mathrm{EMG}_{\mathrm{MRFD}}$ for each condition.

\begin{tabular}{|c|c|c|c|c|c|c|c|}
\hline & CV\% & nCM & nVE & VE & $F(2.44)$ & $\eta^{2}$ & $P$ \\
\hline \multirow{2}{*}{ FDS } & $\mathrm{EMG}_{\mathrm{MVF}}$ & $19.88 \pm 9.44$ & $12.39 \pm 12.52 *$ & $11.77 \pm 8.01 *$ & 4.826 & 0.18 & 0.013 \\
\hline & $\mathrm{EMG}_{\mathrm{MRFD}}$ & $23.06 \pm 13.34$ & $14.23 \pm 9.35^{*}$ & $15.17 \pm 13.83^{*}$ & 4.337 & 0.16 & 0.019 \\
\hline \multirow{2}{*}{ EDC } & $\mathrm{EMG}_{\mathrm{MVF}}$ & $16.18 \pm 7.27$ & $11.11 \pm 5.73 \#$ & $8.40 \pm 4.67 \S$ & & 0.37 & $<0.001$ \\
\hline & $\mathrm{EMG}_{\mathrm{MRFD}}$ & $22.87 \pm 7.79 \mathrm{~A}$ & $11.83 \pm 8.82 \S$ & $10.43 \pm 10.43 \xi$ & 17.871 & 0.45 & $<0.001$ \\
\hline
\end{tabular}

Verbal encouragement (VE), non verbal encouragement (nVE), non concentration and motivation (nCM)

* $(\mathrm{p}<0.05), \#(\mathrm{p}<0.01), \S(\mathrm{p}<0.001)$ significantly lower compared with nCM

$\$(\mathrm{p}<0.001)$ significantly higher compared with $\mathrm{nVE}$

$¥(\mathrm{p}<0.001)$ significantly higher compared with VE

A $(p<0.05)$ significantly higher compared with CV\% MVF

Table 4: Standardized differences between instructions and rating higher/similar/lower

\begin{tabular}{|c|c|c|c|c|c|}
\hline \multirow{2}{*}{ Variable } & \multirow{2}{*}{$\begin{array}{c}\text { nVE compared with nCM } \\
\text { ES }(95 \% \mathrm{CD}) \quad \text { Rating }\end{array}$} & \multirow{2}{*}{$\begin{array}{c}\text { VE compared with nCM } \\
\text { ES }(95 \% \mathrm{CI})\end{array}$} & \multicolumn{3}{|c|}{ VE compared with nVE } \\
\hline & & & Rating & ES $(95 \% \mathrm{CI})$ & Rating \\
\hline MVF & $0.17(0.13$ to 0.21$)$ Trivial & $0.42(0.31$ to 0.53$)$ & Small & $0.25(0.16$ to 0.35$)$ & Small \\
\hline $\operatorname{MRFD}\left(\mathrm{kg} \cdot \mathrm{s}^{-1}\right)$ & $0.29(0.18$ to 0.40$) \quad$ Small & $0.67(0.48$ to 0.86$)$ & Medium & $0.38(0.22$ to 0.54$)$ & Small \\
\hline MRFD \% $\left(. s^{-1}\right)$ & $0.35(0.11$ to 0.58$)$ & $0.64(0.31$ to 0.98$)$ & Medium & $0.30(0.05$ to 0.54$)$ & Small \\
\hline iEMGMVF & $0.85(0.49$ to 1.22$)$ & $1.79(1.27$ to 2.30$)$ & Large & $0.94(0.63$ to 1.24$)$ & Large \\
\hline iEMG ${ }_{M R F D}$ & $0.79(0.51$ to 1.08$)$ & $1.94(1.63$ to 2.25$)$ & Large & $1.15(0.87$ to 1.43$)$ & Large \\
\hline
\end{tabular}

